\title{
The fast recovery dynamics of a quantum dot semiconductor optical amplifier
}

\author{
Thomas Erneux, ${ }^{1}$ Evgeny A. Viktorov ${ }_{3}^{1}$ Paul Mandel, ${ }^{1, a)}$ Tomasz Piwonski, ${ }^{2}$ \\ Guillaume Huyet, ${ }^{2}$ and John Houlihan ${ }^{3}$ \\ ${ }^{1}$ Optique Nonlinéaire Théorique, Université Libre de Bruxelles, Campus Plaine, Code Postal 231, 1050 \\ Bruxelles, Belgium \\ ${ }^{2}$ Tyndall National Institute, Cork, Ireland, and Department of Applied Physics, Cork Institute of Technology, \\ Cork, Ireland \\ ${ }^{3}$ Department of Computing Maths and Physics, Waterford Institute of Technology, Waterford, Ireland
}

(Received 3 February 2009; accepted 22 February 2009; published online 16 March 2009)

\begin{abstract}
We consider a rate equation model of a quantum dot semiconductor optical amplifier that takes into account carrier capture, escape, and Pauli blocking processes. We evaluate possible differences between phonon-assisted or Auger processes being dominant for recovery. An analytical solution which corresponds to phonon-assisted interaction is then used to accurately fit experimental recovery curves and allows an estimation of both the carrier capture and escape rates. (C) 2009 American Institute of Physics. [DOI: 10.1063/1.3098361]
\end{abstract}

Quantum dot (QD) semiconductor optical amplifiers (SOAs) demonstrate faster gain recovery ${ }^{1}$ and a suppression of pattern effects ${ }^{2}$ when compared with SOAs based on bulk or quantum well materials. As a result, QD SOAs are very promising for applications in high speed optical communications. One of the most important features of QD materials is the discrete structure of their energy levels; the speed of carrier transfer between these levels provides a natural limitation on the bandwidth of QD SOAs. The study of carrier dynamics, based on ultrafast spectroscopy, may thus lead to new ways to optimize QD devices. The first pump-probe experiments have shown that the recovery dynamics in SOAs exhibits several time scales, which can be roughly characterized as ultrafast $(\lesssim 1 \mathrm{ps})$, fast (10 ps), and slow $(>100 \mathrm{ps}) .^{3-6}$ The ultrafast and fast scales are commonly attributed to the intradot relaxations and to the dot refilling by capture from the wetting layer. The slow scale relates to interband recombination between electrons and holes.

In Refs. 7 and 8 recovery time scales are extracted from the data using a multiexponential fitting procedure. While useful for identifying the limiting time scales of SOAs, such approaches have not been able to extract time scales for higher order processes such as thermalization of carriers from lower to higher energy levels, and carriers from the dot to the wetting layer. In this letter, we propose a model-based investigation of the QD SOA fast scale dynamics (10 ps range) that takes into account capture and escape processes. We determine an analytical solution for the nonlinear recovery dynamics that is then used as a fitting function to extract fundamental time constants from differential transmission measurements of InAs-based QDs. The computed capture and escape times agree well with published values and do not change significantly over a broad range of bias values, as expected. Because we use a single nonlinear function derived from a physical model rather than multiple exponential functions, our method allows us to extract rate constants directly from the data. Our general approach is based on rate equa-

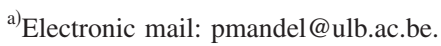

tions, and facillitates the study of a wide range of SOAs by appropriate modifications in the gain model.

The experiment is a single color heterodyne pump-probe arrangement applied to a QD SOA. Details on the setup and device can be found in Ref. 3. We analyze the experimental recovery time traces with a single exponential fitting $\rho=a$

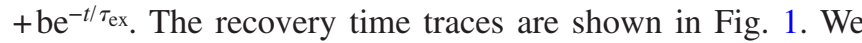
find $\tau_{\mathrm{ex}}=3-4$ ps over a wide current range. Figure 1 indicates that the recovery rate $\tau_{\mathrm{ex}}^{-1}$ is a linear function of the pump current. Note that the fits obtained from the experimental data suggest that the recovery dynamics depend on a single carrier relaxation time. However, by assuming both capture and escape processes we will show that this is not the case and extract these two relaxation times.

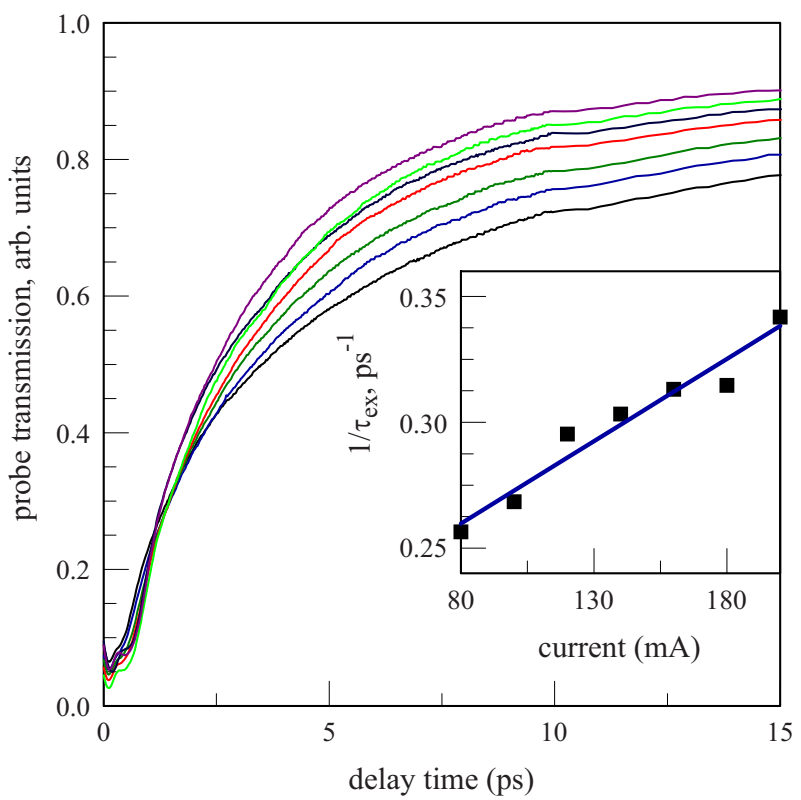

FIG. 1. (Color online) Example of an experimental time traces. Inset: carrier capture rates $\tau_{\mathrm{ex}}^{-1}$ extracted from single exponential fits of the experimental data over a wide current range (squares). Note that the values are linearly dependent of current (bold line). 
Following Ref. 9, we introduce the rate equations for the occupation probability $\rho$ of the dot and the number $n$ of carriers in the wetting layer per dot,

$$
\begin{aligned}
& \partial \rho / \partial t \equiv \rho^{\prime}=-\tau^{-1} \rho+F(\rho, n), \\
& n^{\prime}=\tau^{-1}(J-n)-2 F(\rho, n) .
\end{aligned}
$$

The factor 2 in Eq. (2) accounts for the spin degeneracy in the QD energy levels. The parameter $\tau$ is the carrier recombination time, assumed the same for the wetting layer and the dots for mathematical simplicity. $J$ is the pump current per dot. The function $F(\rho, n)$ describes the carrier exchange rate between the wetting layer and the dots. In its most general form, it can be expressed as

$$
F(\rho, n)=R^{\mathrm{cap}}(1-\rho)-R^{\mathrm{esc}} \rho,
$$

where $1-\rho$ is the Pauli blocking factor and $R^{\text {cap }}(n)$ and $R^{\text {esc }}(n)$ are defined as the carrier capture and escape rates, respectively. For the capture process, we consider $R^{\text {cap }}$ $=\tau_{\text {cap }}^{-1} n$, where $\tau_{\text {cap }}$ is defined as the dot capture time and is an intrinsic property of the dot (phonon-assisted interaction). Alternatively, the effect of more complicated processes may be investigated, as in Ref. 3, where $R^{\text {cap }}=\tau_{\text {cap }}^{-1} n^{2}$ models the Auger-type carrier relaxation. We shall analyze both cases. On the other hand, the carrier escape rate is assumed constant, $R^{\text {esc }}=\tau_{\text {ecs }}^{-1}$, where $\tau_{\text {esc }}$ is the carrier escape time from the dot to the wetting layer at room temperature.

Equations (1) and (2) describe the time-dependent recovery of the QD SOA. Its solution depends on the four parameters $\tau_{\text {cap }}, \tau_{\text {esc }}, \tau$, and $J$, and the initial condition $\rho(0)=\rho_{0}$ $<1$ with $n(0)=n_{0} \neq 0$ which are themselves functions of the pump current. To limit the analysis, we assume that $\tau$ is much larger than $\tau_{\text {cap }}$ and $\tau_{\text {esc }}$, and that $\rho_{0}$ and $n_{0}$ increase linearly with $J$. Our model neglects the effects of intradot relaxation introduced in Refs. 4, 6, and 10, however these occur over times of $<1 \mathrm{ps}$ and their omission allows an analytical understanding of the slower timescales, i.e., the 10 ps range.

The analytical solution will be used as a fitting function for the experimental data with the purpose to determine both $\tau_{\text {cap }}$ and $\tau_{\text {esc }}$. Note that the usual fitting procedure yield values for the rate $R^{\text {cap }}$ rather than the intrinsic capture rate constant $\tau_{\text {cap. }}$. Moreover, $\tau_{\text {esc }}$ is usually inaccessible by this method.

Experimentally, an ultrafast stage precedes the fast recovery studied here. It is attributed to a mix of intradot dynamics, second harmonic generation, and interactions between pump and probe pulses (coherent effects). As none of these processes are considered in the model, the data obtained during the first picoseconds are neglected. ${ }^{11}$

Adding twice, Eqs. (1) and (2) leads to a linear equation that admits the solution

$$
2 \rho+n=\left(2 \rho_{0}+n_{0}-J\right) \exp (-t / \tau)+J .
$$

Assuming now that $t$ is of the order of either $\tau_{\text {cap }}$ or $\tau_{\text {esc }}$, the exponential in (4) remains close to $1\left(\tau \gg \tau_{\text {cap }}, \tau_{\text {esc }}\right)$ and the expression (4) is simplified as

$$
2 \rho+n=2 \rho_{0}+n_{0} .
$$

Using Eq. (5), we determine $n$ as a function of $\rho$ and eliminate $n$ in Eq. (1). The resulting equation for $\rho$ is

$$
\rho^{\prime}=\tau_{\text {cap }}^{-1}\left(2 \rho_{0}+n_{0}-2 \rho\right)^{\alpha}(1-\rho)-\left(\tau^{-1}+\tau_{\text {esc }}^{-1}\right) \rho,
$$

where $\alpha=1$ or 2 corresponding to either a phonon-assisted or an Auger-type relaxation process.

The problem now depends on two parameters: the initial number of carriers $2 \rho_{0}+n_{0}$ and the relative speed of the energy exchange between the dot and the wetting layer determined by the ratio $\varepsilon=\tau_{\text {cap }} / \tau_{\text {esc }}$. The number of carriers $2 \rho_{0}$ $+n_{0}>2$ depends on the pump current. The ratio $\varepsilon$ can be estimated via quasi-Fermi equilibrium condition $^{12}$ which gives $\varepsilon \ll 1$.

With these conditions, we find that $\rho \lessgtr 1$ is the only stable steady state solution of Eq. (6). In order to evaluate possible differences between phonon-assisted and Auger processes, it is instructive to determine the exponential decay rate toward this steady state. We find the decay rates

$$
\begin{gathered}
\Gamma_{\alpha=1} \equiv \Gamma_{1}=\left(2 \rho_{0}+n_{0}-2\right)+O(\varepsilon), \\
\Gamma_{\alpha=2} \equiv \Gamma_{2}=\left(2 \rho_{0}+n_{0}-2\right)^{2}+O(\varepsilon)
\end{gathered}
$$

are the decay rates for the phonon-assisted and Auger processes, respectively. Since we assumed that $\rho_{0}$ and $n_{0}$ change linearly with $J$, we then conclude from Eq. (7) that $\Gamma_{1}$ and $\Gamma_{2}$ are linear and quadratic functions of $J$, respectively. However, the results of the exponential fitting in the inset of Fig. 1 suggest a linear dependence on $J$ meaning that phononassisted processes are dominant in our QD SOA.

Equation (6) with $\alpha=1$ (dominant phonon-assisted processes) is separable and admits the solution

$$
\begin{aligned}
& \rho=\frac{\rho_{-}\left(\rho_{+}-\rho_{0}\right)-\rho_{+}\left(\rho_{-}-\rho_{0}\right) \exp \left(-F t / \tau_{\text {cap }}\right)}{\rho_{+}-\rho_{0}-\left(\rho_{-}-\rho_{0}\right) \exp \left(-F t / \tau_{\text {cap }}\right)}, \\
& \rho_{ \pm} \equiv \frac{2 \rho_{0}+n_{0}+2+\varepsilon \pm F}{4}, \\
& F \equiv \sqrt{\left(2 \rho_{0}+n_{0}-2\right)^{2}+2 \varepsilon\left(2 \rho_{0}+n_{0}+2\right)+\varepsilon^{2}} .
\end{aligned}
$$

The solution (8) describes the recovery of the QD SOA as a monotone nonlinear evolution toward $\rho=\rho_{-}$. This evolution clearly depends on the decay rate of the exponential functions given by $\Gamma=F / \tau_{\text {cap }}$ which is a linear function of initial number of carriers $2 \rho_{0}+n_{0}$ for $\varepsilon$ small. Since we assumed $2 \rho_{0}+n_{0}$ to be proportional to the pump current, the decay rate is a linear function of $J$ in the limit $\varepsilon$ small. However, the exact expression for $\Gamma$ is a nonlinear function of both $2 \rho_{0}+n_{0}$ and $\varepsilon$, and we shall use the exact expression (8) to investigate the experimental data. To this end, we fix $\tau$ $=10^{3} \mathrm{ps}$ and consider $\tau_{\text {cap }}, \tau_{\text {esc }}$, and $2 \rho_{0}+n_{0}$ as fitting parameters when we match experimental and theoretical curves. A sample recovery time trace is shown in Fig. 2(a) with the designated area that we have used for fitting. For $\tau_{\text {cap }}$ and $\tau_{\text {esc }}$, the results of fitting over a wide current range are shown on Fig. 2(b) and are largely independent of the current. The resulting mean values are

$$
\tau_{\text {cap }} \simeq 9 \pm 0.75 \mathrm{ps} \text { and } \tau_{\text {esc }} \simeq 80 \pm 10 \mathrm{ps},
$$

and the ratio $\tau_{\text {cap }} / \tau_{\text {esc }} \approx 0.1$ correctly corresponds to the estimates provided by the quasi-Fermi equilibrium. ${ }^{12}$

By contrast to the conventional multiexponential fitting procedure, using the nonlinear evolution function in Eq. (8) offers several advantages. First, it corresponds to the short time solution of a rate equation model for the relaxation of 

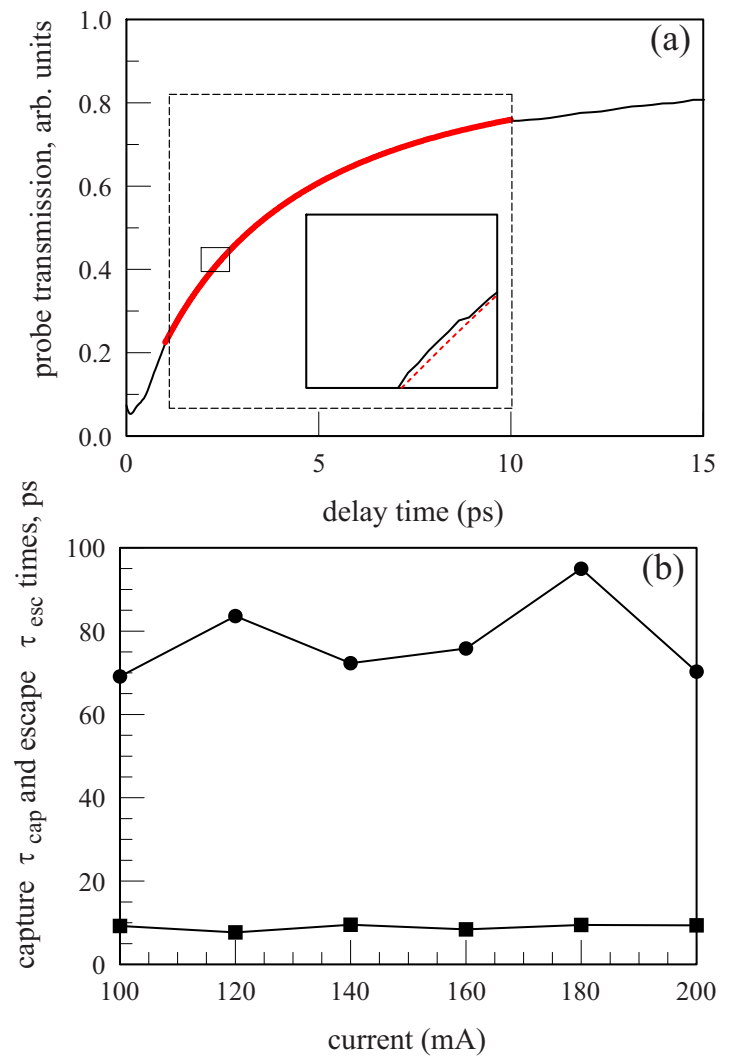

FIG. 2. (Color online) (a) Example of an experimental (black) and fitted with expression (8) (red) gain recovery. The fitting range is indicated by a dashed square and the inset contains a zoomed part of the fitted region $\left(2 \rho_{0}+n_{0}=2.6, \rho_{-}-\rho_{0}=1.7\left(\rho_{+}-\rho_{0}\right), \tau_{\text {cap }}=9.22 \mathrm{ps}\right.$, and $\left.\tau_{\text {esc }}=69.13 \mathrm{ps}\right)$. The experimental current was $100 \mathrm{~mA}$ (transparency at $15 \mathrm{~mA}$ ). (b) Carrier capture and escape times extracted from fits of the experimental data over a wide current range. Note that the values are largely independent of current.

the QD SOA. Other contributions to the relaxation process could be included as we have shown by considering separately phonon-assisted and Auger processes. The function in Eq. (8) is a highly nonlinear function of time and reduces to a single exponential only for large times. It is therefore not surprising that the computed capture time $\tau_{\text {cap }} \simeq 9$ ps is different from the one proposed by the exponential fitting in
Fig. 1. The large ratio $\tau_{\text {cap }} / \tau_{\text {ex }} \approx 3$ emphasizes the fact that we may not underestimate the nonlinear character of the escape process. In addition, our analysis provides a way to estimate both $\tau_{\text {cap }}$ and $\tau_{\text {esc }}$ from the experimental data. The estimate of the escape time does not need the assumption of thermal equilibrium and directly follows from the rate equations describing a system far from equilibrium.

In summary, we have analyzed the fast (1-10 ps) stage of the recovery dynamics in a QD SOA and concentrated on the role of the capture and escape rates. Our analysis provides a nonlinear evolution function for the recovery of the occupation probability of the dot that fits very well the experimental data. We obtained estimates of the capture and escape times which are in the range of 9 and $80 \mathrm{ps,}$ respectively.

The authors acknowledge support of the Fonds National de la Recherche Scientifique (Belgium), Science Foundation Ireland (SFI) under Contract No. 07/IN.1/1929, the Tyndall National Access Programme and the Irish Higher Education Authority under the PRTLI program.

${ }^{1}$ D. Bimberg, J. Phys. D 38, 2055 (2005).

${ }^{2}$ T. Akiyama, N. Hatori, Y. Nakata, H. Ebe, and M. Sugawara, Electron. Lett. 38, 1139 (2002)

${ }^{3}$ T. Piwonski, I. O’Driscoll, J. Houlihan, G. Huyet, R. J. Manning, and A. V. Uskov, Appl. Phys. Lett. 90, 122108 (2007).

${ }^{4}$ T. W. Berg, S. Bischoff, I. Magnusdottir, and J. Mork, IEEE Photonics Technol. Lett. 13, 541 (2001).

${ }^{5}$ M. van der Poel, J. Mork, A. Somers, A. Forchel, J. P. Reithmaier, and G. Eisenstein, Appl. Phys. Lett. 89, 081102 (2006).

${ }^{6}$ I. O'Driscoll, T. Piwonski, C. F. Schleussner, J. Houlihan, G. Huyet, and R. J. Manning, Appl. Phys. Lett. 91, 071111 (2007)

${ }^{7}$ E. U. Rafailov, S. J. White, A. A. Lagatsky, A. Miller, W. Sibbett, D. A. Livshits, A. E. Zhukov, and V. M. Ustinov, IEEE Photonics Technol. Lett. 16, 2439 (2004).

${ }^{8}$ V. Cesari, W. Langbein, P. Borri, M. Rossetti, A. Fiore, S. Mikhrin, I. Krestnikov, and A. Kovsh, Appl. Phys. Lett. 90, 201103 (2007).

${ }^{9}$ Th. Erneux, E. A. Viktorov, and P. Mandel, Phys. Rev. A 76, 023819 (2007).

${ }^{10}$ E. A. Viktorov, P. Mandel, Y. Tanguy, J. Houlihan, and G. Huyet, Appl. Phys. Lett. 87, 053113 (2005).

${ }^{11}$ P. Borri, F. Romstad, W. Langbein, A. E. Kelly, J. Mork, and J. M. Hvam, Opt. Express 7, 107 (2000).

${ }^{12}$ A. Markus, J. X. Chen, O. Gauthier-Lafaye, J.-G. Provost, C. Paranthoën, and A. Fiore, IEEE J. Sel. Top. Quantum Electron. 9, 1308 (2003). 